Physics

Physics Research Publications

Piezoelectric coupling, phonons, and tunneling into a quantum Hall edge

S. Khlebnikov 


\title{
Piezoelectric coupling, phonons, and tunneling into a quantum Hall edge
}

\author{
S. Khlebnikov \\ Department of Physics, Purdue University, West Lafayette, Indiana 47907, USA
}

(Received 10 October 2005; published 30 January 2006)

\begin{abstract}
We show that the piezoelectric coupling to three-dimensional phonons in GaAs renormalizes the currentvoltage exponent for tunneling of electrons into an incompressible quantum Hall edge. The leading correction is always negative, in agreement with experiments on the $\nu=1 / 3$ state, and, depending on the precise value of the edge plasmon speed, can be as large as a few percent. We also discuss higher-order corrections, which determine the effect of the piezoelectric coupling in the extreme infrared limit.
\end{abstract}

DOI: 10.1103/PhysRevB.73.045331

PACS number(s): 73.43.Jn, 71.10.Pm, 71.38.-k

\section{INTRODUCTION}

The discrepancy between the tunneling exponent predicted by the conformal field theory ${ }^{1}$ (CFT) of a quantum Hall $(\mathrm{QH})$ edge and the one measured in cleaved-edgeovergrowth (CEO) experiments, ${ }^{2,3}$ (for a review see Ref. 4) remains a puzzle. For the principal filling fraction $\nu=1 / 3$, the discrepancy is not that large but is still believed to require an explanation (for recent discussions of the issue, see Refs. 5 and 6). A natural way to resolve the discrepancy would be to identify additional gapless modes, not present in the original chiral CFT. Such modes appear, for example, in scenarios based on "edge reconstruction." 7,8 Another group of explanations involves the role of the Coulomb interaction in the presence of a "hard" edge ${ }^{9,10}$ profiles of the electron density obtained numerically in this case are rather similar to those resulting from edge reconstruction. Finally, although we do not address that case in the present paper, we note that there seems to be a discrepancy between theory and experiment also for compressible QH states; for more detail, see Refs. 4 and 11.

The effect of a one-dimensional (1D) "phononlike" mode on scattering between two QH edges was considered in Ref. 12 , and it was shown that a derivative coupling of the charge density to such a mode does renormalize the resistance. For tunneling from a bulk metal into a single edge, as in the CEO experiments, this coupling will similarly renormalize the tunneling exponent. The requisite 1D phononlike mode, propagating only along the edge, can be a result of the edge reconstruction or other mechanisms enumerated in Ref. 12, all of which have to do with the near-edge properties of the electron density.

For the purpose of explaining the experimental data, however, one does not have to consider the electronic system alone. In particular, in the present paper we note a hitherto apparently unidentified effect of the ordinary 3D acoustic phonons in GaAs. The derivative coupling of the type considered in Ref. 12 is irrelevant in this case, in the sense that it does not lead to a logarithmic correction to the propagator of the edge plasmon. (The replacement of the 3D phonon with a $1 \mathrm{D}$ phonon in this problem in Ref. 13 looks to us completely arbitrary.) As we show here, however, there is a logarithmic effect (and consequently a renormalization of the tunneling exponent) due to the piezoelectric coupling.

We limit ourselves to the principal filling fractions $\nu=1 /(2 p+1)$ and assume that the CFT contains a single chi- ral boson-the left-moving edge plasmon mode. ${ }^{1}$ However, the phonon-induced correlation to which we attribute our results may also play a role in a much broader class of problems involving $1 \mathrm{D}$ conductors.

The piezoelectric electron-phonon interaction is described by the Hamiltonian

$$
H_{\text {piezo }}=h \partial_{x} \theta_{L} \phi \delta(y) \delta(z) .
$$

Here $\theta_{L}$ is the chiral field of the edge plasmon, $(1 / 2 \pi) \partial_{x} \theta_{L}$ is the representation of the electron density in the edge CFT, $\phi$ is the phonon field, and $h$ is the coupling constant. The $\delta$ functions restrict the coupling to one dimension, and indeed $\theta_{L}$ is a 1D field; however, $\phi$ is fully three dimensional.

The piezoelectric coupling is known to be highly anisotropic, but here we follow a common practice and use a direction average. The main result - a renormalization of the tunneling exponent-does not depend on this replacement. In fact, one could get rid of the averaging directly in the solution, Eq. (21) below, by bringing $h^{2}$ under the integral over $k_{\perp}$ (the transverse wave number of the phonon field) and supplying it with an angular dependence. This would not affect the structure of the infrared-sensitive terms.

We keep in mind that there are three polarizations of acoustic phonons (two transverse and one longitudinal), but in our calculation their contributions simply add up, so we will think of $\phi$ as representing just one of them. Interactions of the form (1) have been considered in a variety of problems concerning GaAs structures (for recent work, see Ref. 14) but, to our knowledge, not in connection with tunneling into a $\mathrm{QH}$ edge.

It is straightforward to count the powers of momenta and see that the Hamiltonian (1) allows for a logarithmic correction to the propagator of $\theta_{L}$. We can anticipate the nature of the effect by noting that the Hamiltonian gives rise to a nonlocal time-dependent "potential" between electrons, which to the leading order in $h^{2}$ has the form

$$
U(x, \tau)=-\frac{h^{2}}{v_{\mathrm{ph}}\left(x^{2}+v_{\mathrm{ph}}^{2} \tau^{2}\right)} .
$$

Here $v_{\mathrm{ph}}$ is the phonon speed and $\tau$ the Euclidean time. Since this "potential" is attractive, we expect that it will tend to confine the electron cloud near the tunneling site, thus leading to a decrease in the tunneling exponent $\alpha$. 
What remains, then, is to compute, using the interaction Hamiltonian (1), the coefficient of the logarithm, to see if the effect can be large enough to be experimentally accessible. In Sec. III, we will see that it can. The precise answer depends on the ratio $c_{-} / v_{\mathrm{ph}}$ of the speed of the chiral mode to that of the phonon. For $c_{-}$in the range $10^{5}-10^{6} \mathrm{~cm} / \mathrm{s}$, the leading (in $h^{2}$ ) correction to the tunneling exponent ranges from an accessible $\Delta \alpha=-0.09$ to an apparently unobservable $\Delta \alpha=-0.003$. The correction is always negative, in agreement with experiments ${ }^{2,3}$ on the $\nu=1 / 3$ state. Higher-order corrections and the $\nu=1$ state are discussed in Sec. IV. Section V is a summary of the results.

\section{MODEL AND THE PATH INTEGRAL}

We start with the Euclidean action that consists of three parts: the individual actions of the 1D chiral boson $\theta_{L}$ and the 3D phonon $\phi$ and the interaction corresponding to the Hamiltonian (1):

$$
S_{E}=S_{\theta}+S_{\phi}+\int d \tau d^{3} x H_{\text {piezo }} .
$$

A term that describes tunneling of a single electron between a Fermi liquid and the QH edge will be added later. In Eq. (3),

$$
\begin{aligned}
& S_{\theta}=\kappa \int d \tau d x\left[-i \partial_{\tau} \theta_{L} \partial_{x} \theta_{L}+c_{-}\left(\partial_{x} \theta_{L}\right)^{2}\right], \\
& S_{\phi}=\int d \tau d^{3} x\left\{\frac{1}{2}\left(\partial_{\tau} \phi\right)^{2}+\frac{1}{2} v_{\mathrm{ph}}^{2}\left(\partial_{x} \phi\right)^{2}\right\},
\end{aligned}
$$

$\kappa=1 / 4 \pi \nu$, and $\tau=i t$ is the Euclidean time. We denote 1D integrations by $d x$ and 3D ones by $d^{3} x$. Our normalization of the field $\theta_{L}$ is such that the expansion in terms of the canonically normalized creation and annihilation operators has the form

$$
\theta_{L}=\sum_{k<0} \frac{1}{\sqrt{2 \kappa|k| L}}\left(b_{k} e^{-i \omega_{-} t+i k x}+b_{k}^{\dagger} e^{i \omega_{-} t-i k x}\right)+\text { z.m. },
$$

where $L$ is the length of the edge and z.m. stands for the zero modes. ${ }^{1}$ In this normalization, the fermion creation operator in the chiral CFT is

$$
\psi^{\dagger}=: \exp \left(\frac{i}{\nu} \theta_{L}\right):
$$

The easiest way to obtain a path integral for the chiral field $\theta_{L}$ is to separate it into even and odd (with respect to $x$ ) components: $\theta_{L}=\theta_{e}+\theta_{o}$. If we consider $\theta_{e}$ as the canonical coordinate, then it follows from Eq. (4) that

$$
p=2 \kappa \partial_{x} \theta_{o}
$$

is the corresponding canonical momentum. The path-integral measure can be written as $\mathcal{D} \theta_{e} \mathcal{D} p$ or, since the Jacobian of transformation from $p$ to $\theta_{o}$ does not depend on the field, equivalently as $\mathcal{D} \theta_{e} \mathcal{D} \theta_{o}=\mathcal{D} \theta_{L}$. Thus, the vacuum-to-vacuum amplitude in the presence of a current $J(x, \tau)$ can be written as

$$
Z[J]=\int \mathcal{D} \theta_{L} \exp \left(-S_{E}+\int d \tau d x J \theta_{L}\right) .
$$

We assume that tunneling occurs at isolated sitesimpurities, or tunneling centers-and in what follows consider just one such site, located at $x=0$. The tunneling term $S_{t}$ is then

$$
S_{t}=\text { const } \int d \tau\left[c(0, \tau) \psi^{\dagger}(0, \tau)+\text { H.c. }\right],
$$

where the operator $c(0, \tau)$ destroys a spin-polarized Fermiliquid electron at point $x=0$. Treating $S_{t}$ perturbatively, we can obtain the tunneling rate via a version of the optical theorem from the two-point correlator of $\psi$. The requisite correlator is given by Eq. (9) with a special choice of current:

$$
J(x, \tau)=\frac{i}{\nu}\left[\delta\left(x-x_{1}\right) \delta\left(\tau-\tau_{1}\right)-\delta\left(x-x_{2}\right) \delta\left(\tau-\tau_{2}\right)\right],
$$

corresponding to insertion of a fermion at $x=x_{1}, \tau=\tau_{1}$ and removal of a fermion at $x=x_{2}, \tau=\tau_{2}$. For the present purposes, we only need to consider $x_{1}=x_{2}=0$, but the structure of the correlator is elucidated by taking general $x_{1}$ and $x_{2}$, so we compute it for that more general case.

Note that the correlator just defined corresponds to the bosonic version of time ordering,

$$
\mathcal{G}(x, \tau)=\left\langle 0\left|T_{B}\left[\psi\left(x_{2}, \tau_{2}\right) \psi^{\dagger}\left(x_{1}, \tau_{1}\right)\right]\right| 0\right\rangle,
$$

where $x=x_{2}-x_{1}, \tau=\tau_{2}-\tau_{1}$, and

$$
T_{B}\left[A\left(\tau_{2}\right) B\left(\tau_{1}\right)\right]=\Theta(\tau) A\left(\tau_{2}\right) B\left(\tau_{1}\right)+\Theta(-\tau) B\left(\tau_{1}\right) A\left(\tau_{2}\right),
$$

even though the exponentials (7) are in fact fermions. This is inconsequential since the correlator (12) does not occur as an internal line in any Feynman diagram.

We consider the case of zero temperature, $T=0$. The optical theorem gives the tunneling rate per a unit interval of the biasing energy $E>0$ in terms of the analytical continuation of $\mathcal{G}(x, \tau)$ to real time. For tunneling into the edge, the rate is

$$
\frac{d \mathcal{R}}{d E} \propto N(-E) \operatorname{Im}\left[i \int_{0}^{\infty} d t e^{i E t} \mathcal{G}(0, i t+\delta)\right],
$$

where $N(-E)$ is the density of states in the Fermi liquid. As indicated by the infinitesimal $\delta>0$, in Eq. (14) we need the values of the correlator just below the real- $t$ axis. These can be obtained by analytically continuing the Euclidean $\mathcal{G}(x, \tau)$ from $\tau>0$.

Because the theory (3) is Gaussian, the path integral (9) can be computed exactly:

$$
Z[J]=\exp \left(\frac{1}{2} J G J\right),
$$

where $G$ is the full Green function of the chiral boson $\theta_{L}$ [convolution integrals are implied in the exponent of Eq. 
(15)]. In the absence of phonons, $G$ can be replaced by the free Green function

$$
G_{0}(x, \tau)=-i \nu \int \frac{d \Omega d k_{x}}{2 \pi} \frac{e^{-i \Omega \tau+i k_{x} x}}{k_{x}\left(\Omega-i c_{-} k_{x}\right)},
$$

which to logarithmic accuracy equals

$$
G_{0}(x, \tau)=\left\{\begin{array}{cl}
\nu \ln \frac{L}{c_{-} \tau+i x}, & \tau>0, \\
\nu \ln \frac{L}{-c_{-} \tau-i x}, & \tau<0 .
\end{array}\right.
$$

$L$ is an infrared cutoff, any dependence on which will disappear when we use $G_{0}$ (in place of $G$ ) in Eq. (15).

At small $E$, where $N(-E)$ tends to a constant, Eq. (14) then becomes

$$
\frac{d \mathcal{R}}{d E} \propto \operatorname{Im}\left[i \int_{0}^{\infty} d t e^{i E t}(i t+\delta)^{-\alpha}\right]=\frac{\pi}{\Gamma(\alpha)} E^{\alpha-1},
$$

where $\alpha=4 \pi \kappa=1 / \nu$. The rate at a fixed biasing voltage $V$ is

$$
\mathcal{R}=\int_{0}^{e V} \frac{d \mathcal{R}}{d E} d E \propto V^{\alpha} .
$$

Thus, in the model without phonons, the tunneling exponent is $\alpha=1 / \nu$ - the prediction of the chiral CFT. ${ }^{1}$

\section{CORRECTION TO THE TUNNELING EXPONENT}

We are interested in corrections to the results (18) and (19) due to the piezoelectric coupling (1). The full Green function $G$ of the chiral boson is given by the Fourier transform

$$
G(x, \tau)=\int \frac{d \Omega d k_{x}}{(2 \pi)^{2}} e^{-i \Omega \tau+i k_{x} x} F\left(\Omega, k_{x}\right),
$$

where

$$
F^{-1}=2 \kappa k_{x}\left(i \Omega+c_{-} k_{x}\right)-h^{2} k_{x}^{2} \int \frac{d^{2} k_{\perp}}{(2 \pi)^{2}} \frac{1}{\Omega^{2}+v_{\mathrm{ph}}^{2} k^{2}},
$$

$k_{\perp}=\left(k_{y}, k_{z}\right)$, and $k^{2}=k_{x}^{2}+k_{\perp}^{2}$. Note that it is the coefficient of $\ln \tau$ in the boson Green function (at $x=0$ ) that determines the tunneling exponent $\alpha$. Consequently, we are interested in logarithmic contributions to $G$.

Formally, Eq. (20) is the exact solution to our problem. However, it is not easy to analyze. Alternatively, it can be used to obtain a perturbative expansion of $G$-i.e., an expansion in powers of $h^{2}$ :

$$
G(x, \tau)=G_{0}(x, \tau)+G_{1}(x, \tau)+\cdots .
$$

The leading term is the free propagator (16), and the nextto-leading term equals

$$
G_{1}(x, \tau)=-\frac{h^{2}}{4 \kappa^{2}} \int \frac{d^{3} k d \Omega}{(2 \pi)^{4}} \frac{e^{-i \Omega \tau+i k_{x} x}}{\left(\Omega-i c_{-} k_{x}\right)^{2}\left(v_{\mathrm{ph}}^{2} k^{2}+\Omega^{2}\right)} .
$$

Since the coupling $h^{2}$ is relatively small, we first concentrate on this term; effects of the higher-order corrections are discussed in the next section.

Writing

$$
G_{1}(x, \tau)=\frac{h^{2}}{16 \pi^{2} \kappa^{2} v_{\mathrm{ph}}^{2}} I(x, \tau),
$$

we find three types of logarithmic terms in the integral $I$ : $I$ $\approx I_{1}+I_{2}+I_{3}$; for $\tau>0$,

$$
\begin{gathered}
I_{1}=-\frac{\tau}{c_{-} \tau+i x} \ln \left[\Lambda\left(c_{-} \tau+i x\right)\right], \\
I_{2}=\frac{c_{-}}{v_{\mathrm{ph}}^{2}-c_{-}^{2}} \ln \frac{L}{c_{-} \tau+i x}, \\
I_{3}=-\frac{1}{2} \sum_{s= \pm 1} \frac{1}{v_{\mathrm{ph}}+s c_{-}} \ln \frac{L}{v_{\mathrm{ph}} \tau-i s x} .
\end{gathered}
$$

$\Lambda$ is the ultraviolet (momentum) cutoff. The approximate sign in the expression for $I$ means that only terms that give rise to $\ln \tau$ are kept.

Setting $x=0$, we obtain, to logarithmic accuracy,

$$
G_{1}(x, \tau)=\frac{h^{2} \nu^{2}}{v_{\mathrm{ph}} c_{-}\left(v_{\mathrm{ph}}+c_{-}\right)} \ln \tau .
$$

The corresponding correction to the tunneling exponent is

$$
\Delta \alpha=-\frac{2 h^{2}}{v_{\mathrm{ph}} c_{-}\left(v_{\mathrm{ph}}+c_{-}\right)},
$$

where the factor of 2 is due to the presence of two transverse polarizations of the phonon (the third, longitudinal, polarization has larger velocity and gives a smaller contribution). Note that the correction is always negative.

For estimates, we use the same expression for the piezoelectric coupling and the same values of the parameters as in Ref. 14. In our present notation,

$$
h^{2}=\frac{1}{\hbar \rho_{M}}\left(\frac{e e_{14}}{4 \pi \epsilon}\right)^{2},
$$

where $\rho_{M}=5.36 \mathrm{~g} / \mathrm{cm}^{3}, e_{14}=0.16 \mathrm{C} / \mathrm{m}^{2}$, and $\epsilon=13.2 \epsilon_{0}$; in this equation only, we have restored $\hbar$. With these numbers, $h^{2}=5.39 \times 10^{8} \mathrm{~m}^{3} / \mathrm{s}^{3}$. Using also $v_{\mathrm{ph}}=3000 \mathrm{~m} / \mathrm{s}$, we can rewrite Eq. (26) as a function of a single parameter, the ratio

$$
r=c_{-} / v_{\mathrm{ph}} .
$$

We obtain

$$
\Delta \alpha=-\frac{0.04}{r(1+r)} .
$$

Clearly, the smaller is $r$ the larger is the correction. While for $c_{-}=10^{4} \mathrm{~m} / \mathrm{s}$ it is only 0.003 in the absolute value, for 
$c_{-}=10^{3} \mathrm{~m} / \mathrm{s}$ it is already 0.09 . The latter number is roughly of the same order of magnitude as the discrepancy between the CFT prediction $\alpha=3$ for $\nu=1 / 3$ and the central values $\alpha=2.65-2.85$ obtained experimentally ${ }^{2,3}$ for different samples. This number is also larger than the experimental uncertainty quoted for the experiment of Ref. 2.

The most obvious way to experimentally test the present theory is to reduce the plasmon speed via a capacitive coupling to external conductors. In that case, Eq. (29) predicts a further decrease in $\alpha$. This prediction, however, holds only insofar as corrections of higher orders in $h^{2}$ can be neglected; cf. the next section.

\section{HIGHER-ORDER CORRECTIONS}

Expanding the exact Fourier transform (21) in powers of $h^{2}$, we obtain higher-order corrections to the plasmon Green function. In this section, we consider the structure of the perturbation series for the case $c_{-} \ll v_{\mathrm{ph}}$, which is the simplest. In this case, the main contribution to $G$ in a given order can be found by dropping $\Omega^{2}$ in the integral over $k_{\perp}$. The $n$ th-order correction to the Green function at $\tau>0$ becomes

$$
G_{n}(x, \tau) \approx \frac{\nu}{n !}\left(\frac{h^{2} \nu \tau}{v_{\mathrm{ph}}^{2}}\right)^{n} \int_{0}^{\Lambda} d k_{x} k_{x}^{n-1} e^{-\left(c_{-} \tau+i x\right) k_{x}} \ln ^{n} \frac{\Lambda}{k_{x}} .
$$

At $x=0$ all these terms are positive, so their sum remains the main contribution to the sum of the entire perturbation series.

From now on we concentrate on $x=0$. It turns out that the sum of (30) over $n$ saturates at values of $n$ that are much smaller than $n_{0} \equiv \Lambda c_{-} \tau$. For such $n$, the logarithm in (30) can be considered a slowly varying function of $k_{x}$, so that (for $n \neq 0$ )

$$
G_{n}(x, \tau) \approx \frac{\nu}{n}\left(\frac{h^{2} \nu}{v_{\mathrm{ph}}^{2} c_{-}}\right)^{n} \ln ^{n} \frac{n_{0}}{n} \equiv \frac{\nu}{n} \xi^{n} \ln ^{n} \frac{n_{0}}{n} .
$$

The last equality defines the dimensionless coupling $\xi$.

It is somewhat more convenient to consider, instead of the sum of $G_{n}$, the sum of their derivatives with respect to $n_{0}$. Thus, if we denote by $\Delta G$ the full correction to the Green function, $\Delta G=G-G_{0}$, then

$$
\frac{\partial \Delta G}{\partial n_{0}}=\frac{\nu}{n_{0}} \sum_{n=1}^{\left[n_{0}\right]} \xi^{n} \ln ^{n-1} \frac{n_{0}}{n} .
$$

If $n$ is regarded as a continuous variable, the expression under the sum has a maximum at $n=n_{*}$, where

$$
n_{*}=n_{0} e^{-(1+\xi) / \xi}\left[1+O\left(\frac{1}{n_{*}}\right)+O(\xi)\right] .
$$

We define a characteristic time $\tau_{0}$ by

$$
\tau_{0}^{-1}=c_{-} \Lambda \exp \left(-\frac{v_{\mathrm{ph}}^{2} c_{-}}{h^{2} \nu}-1\right) .
$$

Equation (33) can now be written simply as $n_{*} \approx \tau / \tau_{0}$. We see that $\tau_{0}$ is the infrared scale at which the perturbation theory breaks down. At $\tau \ll \tau_{0}$, the sum over $n$ is essentially discrete and is well approximated by the lowest-order term. On the other hand, if $\tau$ is significantly larger than $\tau_{0}$, many terms contribute. In that case, $n$ is indeed quasicontinuous.

At even larger times $\tau \gg \tau_{0} / \xi$, the steepest-descent condition becomes applicable near $n=n_{*}$, and using steepest descent we obtain

$$
\frac{\partial \Delta G}{\partial n_{0}}=\frac{\nu}{n_{0}}\left(2 \pi n_{*} \xi\right)^{1 / 2} \exp \left[\xi \tau / \tau_{0}+O\left(n_{*} \xi^{2}\right)\right] .
$$

The exponential growth of Eq. (35) at large $\tau$ indicates the presence of a plasmon state with energy

$$
E_{p}=-\frac{h^{2} \nu}{v_{\mathrm{ph}}^{2} c_{-} \tau_{0}} .
$$

The same conclusion can be reached by looking directly at the resummed expression (21) or, more precisely, its realtime version obtained by replacing $\Omega$ with $-i \omega$. (In the presence of a state with a negative energy, the rotation to Euclidean frequencies needs to be redefined.) Neglecting again the frequency dependence of the integral over $k_{\perp}$, we see that the interaction shifts the pole of $F$ from $\omega=-c_{-} k_{x}$ to $\omega=f\left(k_{x}\right)$, where

$$
f\left(k_{x}\right)=-c_{-} k_{x}\left(1-\xi \ln \frac{\Lambda}{\left|k_{x}\right|}\right) .
$$

This function has extrema at $k_{x}= \pm\left(c_{-} \tau_{0}\right)^{-1}$, where it equals $\pm\left|E_{p}\right|$.

We interpret the presence of plasmon states with negative energies as a reflection of the polaron effect-formation of a bound state of an electron and the phonon field. This interpretation is supported by the following estimate. Suppose we qualitatively describe the cumulative effect of the attractive interaction (2) over time by the time-independent potential

$$
\tilde{U}(x)=\int U(x, \tau) d \tau=-\frac{\pi h^{2}}{v_{\mathrm{ph}}^{2}|x|} .
$$

For an electron cloud with density distribution $n(x)$ and spatial size of order of the "correlation length" $c_{-} \tau_{0}$, the binding energy can then be estimated as

$$
E_{e}=\frac{1}{2} \int d x d y n(x) \tilde{U}(x-y) n(y) \sim-\frac{h^{2} \ln \left(\Lambda c_{-} \tau_{0}\right)}{v_{\mathrm{ph}}^{2} c_{-} \tau_{0}} .
$$

An electron cloud can be thought of as containing of order $(1 / \nu) \ln \left(\Lambda c_{-} \tau_{0}\right)$ plasmons (in the sense that this is how many plasmons are typically produced when an electron-hole pair annihilates). Dividing Eq. (39) by this number, we obtain an estimate of energy per plasmon in agreement with Eq. (36).

A corollary to this argument is that any infrared effects associated with production of plasmons should saturate at time scales of order $\tau_{0}$ (equivalently, length scales of order $c_{-} \tau_{0}$, the size of the polaron). We therefore expect that in the extreme infrared, at biasing energies 


$$
E \sim\left|E_{e}\right| \sim \frac{1}{\nu \tau_{0}},
$$

the system, at any $\nu$, will cross over to the normal Fermiliquid behavior.

We now estimate the time scale $\tau_{0}$ for $\nu=1 / 3$ and $\nu=1$. The maximal phonon momentum $\Lambda$ is $\Lambda=\omega_{D} / v_{\mathrm{ph}}$, where $\omega_{D}$ is the Debye frequency; for GaAs, $\omega_{D}=345 \mathrm{~K} .{ }^{15}$ Given the uncertainty introduced by the directional averaging of $h$ and by the imprecise knowledge of $r=c_{-} / v_{\mathrm{ph}}$, we prefer to extract the entire combination $y=h^{2} / v_{\mathrm{ph}}^{2} c_{-}$, occurring in Eq. (34), directly from the experimentally measured values of the tunneling exponent. In the limit $c_{-} \ll v_{\mathrm{ph}}$, the lowest-order correction (26) is simply $\Delta \alpha=-2 y$. Assuming (and confirming a posteriori) that the perturbation theory works well for the $\nu=1 / 3$ state, we find that, e.g., the sample ${ }^{2}$ with $\alpha=2.7$ corresponds to $y=0.15$. For $\nu=1 / 3$, Eq. (34) then gives $\tau_{0}^{-1} \sim 10^{-9} r \omega_{D}$, which is unobservably small. We conclude that for $\nu=1 / 3$, in the experimentally relevant range of voltages and temperatures, the lowest-order result (26) is reliable.

The situation is quite different for $\nu=1$. Using the same value of $y$, we now obtain $\tau_{0}^{-1}=14 r \mu \mathrm{eV}$, close to the observable range of energies. The lowest-order result (26) predicts a negative correction to the tunneling exponent at $\nu=1$. Ex- perimentally, no such negative correction has been observed. ${ }^{2,16}$ We see that this may be related to the breakdown of the perturbation theory for $\nu=1$.

\section{CONCLUSION}

We have computed the leading correction [ Eq. (26)], due to the piezoelectric coupling to 3D phonons, to the currentvoltage exponent $\alpha$ for tunneling between a Fermi liquid and a $\nu=1 /(2 p+1) \mathrm{QH}$ edge. The correction is always negative, in agreement with the experiments ${ }^{2,3}$ on the $\nu=1 / 3$ state, and its magnitude depends on the value of the edge plasmon speed.

We have also shown that, in the experimentally relevant range of energies, higher-order corrections for $\nu=1 / 3$ are small and do not invalidate the leading-order result, but for $\nu=1$ they very well might. In neither case, however, the leading-order result (26) represents the true infrared behavior: at a sufficiently low energy, higher-order effects will always become important.

\section{ACKNOWLEDGMENTS}

The author thanks A. Boyarsky, A. Chang, and V. Cheianov for discussions.
${ }^{1}$ X. G. Wen, Phys. Rev. B 41, 12838 (1990); Phys. Rev. B 44, 5708 (1991).

${ }^{2}$ A. M. Chang, L. N. Pfeiffer, and K. W. West, Phys. Rev. Lett. 77, 2538 (1996).

${ }^{3}$ M. Grayson, D. C. Tsui, L. N. Pfeiffer, K. W. West, and A. M. Chang, Phys. Rev. Lett. 80, 1062 (1998).

${ }^{4}$ A. M. Chang, Rev. Mod. Phys. 75, 1449 (2003).

${ }^{5}$ A. Boyarsky, V. V. Cheianov, and O. Ruchayskiy, Phys. Rev. B 70, 235309 (2004).

${ }^{6}$ X. Wan, F. Evers, and E. H. Rezayi, Phys. Rev. Lett. 94, 166804 (2005).

${ }^{7}$ C. de C. Chamon and X. G. Wen, Phys. Rev. B 49, 8227 (1994).

${ }^{8}$ X. Wan, K. Yang and E. H. Rezayi, Phys. Rev. Lett. 88, 056802 (2002).
${ }^{9}$ E. V. Tsiper and V. J. Goldman, Phys. Rev. B 64, 165311 (2001).

${ }^{10}$ S. S. Mandal and J. K. Jain, Solid State Commun. 118, 503 (2001).

${ }^{11}$ L. S. Levitov, A. V. Shytov, and B. I. Halperin, Phys. Rev. B 64, 075322 (2001).

${ }^{12}$ B. Rosenow and B. I. Halperin, Phys. Rev. Lett. 88, 096404 (2002).

${ }^{13}$ O. Heinonen and S. Eggert, Phys. Rev. Lett. 77, 358 (1996).

${ }^{14}$ G. Seelig, K. A. Matveev, and A. V. Andreev, Phys. Rev. Lett. 94, 066802 (2005).

${ }^{15}$ S. Adachi, J. Appl. Phys. 58, R1 (1985).

${ }^{16}$ M. Hilke, D. C. Tsui, M. Grayson, L. N. Pfeiffer, and K. W. West, Phys. Rev. Lett. 87, 186806 (2001). 